\title{
On a Semigroup generated by the Heat Equation with a Nonlinear Neumann Boundary Condition
}

\author{
Ruediger Landes
}

Communicated by Mark Ablowitz, received January 28, 2014.

\begin{abstract}
Recently, a nonlinear boundary value problem of the heat equation has been introduced to describe critical heated boiling regimes. Speetjens et. al. have conjectured that the solution gives rise to semigroup with an attractor being the unstable manifold of its fixed point set. We verify that the solution has indeed that property. But we point out that the semigroup should be considered acting on $L^{2}$ and not on $H^{1}$ as suggested in the conjecture.
\end{abstract}

\section{Contents}

Introduction

Discussion of the conjectured properties in Speetjens et al.

Uniqueness

Existence

\section{Introduction}

In this note we study the $L^{2}$-semigroup generated by the weak solution of the nonlinear boundary value problem

1991 Mathematics Subject Classification. Primary 54C40, 14E20; Secondary 46E25, 20C20.

Key words and phrases. Semigroup, heat equation, Nonlinear Boundary conditions, longtime behavior of boiling regimes.

The author gratefully acknowledges the partial support of Research Council of the University of Oklahoma and of the Center for Dynamical Systems in Dresden, Germany. 


$$
(N P) \begin{cases}u_{t}-\nu \Delta_{(x, y)} u=0, & (t, \xi)=(t, x, y) \in Q_{T}=(0, T) \times \Omega \\ u(0, \xi)=u_{\mathrm{o}}(\xi), & =(0, T) \times\left(B_{R} \times(0, H)\right) \\ -u_{y}(t, x, 0)=0 & \\ u_{y}(t, x, H)=-f(u), & \\ \frac{\partial}{\partial \mathbf{n}} u(t, R \mathbf{n}, y)=0 . & \end{cases}
$$

where $B_{R} \subset \mathbb{R}^{n-1}$ is the $n-1$-dimensional ball with radius $R$, and $f$ is a cubic like function, with its first zero at the origin.

For $n=3$, this nonlinear boundary value problem has been introduced by W. Marquardt at RWTU Aachen to model the interaction between two boiling regimes in highly efficient steam generators and other industrial boiling equipment, c.f. $[\mathrm{AM}]$.

Our study is motivated by the recent work on the long-time behavior of the solutions of the above parabolic problem, c.f.: eg.: [SR], [Sp], [GS]. In order to justify those calculations the authors conjecture that the initial value problem generates a semigroup to which the theory of dynamical systems in infinitely dimensional spaces is applicable as, for instance, presented in Temam's book [T].

In this note we point out that this is indeed the case, but argue that the underlying Hilbert space should be chosen to be $L^{2}$ and not $H^{1}$, as suggested in [SR] c.f. the detailed discussion below.

The author would like to acknowledge the hospitality and support of the Center of Dynamical Systems and the Department of Mathematics at the University of Dresden, Germany and the financial support of the Research Council of the University of Oklahoma. We are grateful for many stimulating discussions with Professors Ralph Chill and Stefan Sigmund, which made this work possible. In particular we owe the crucial reference $[\mathrm{JK}]$ in the proof of the existence of an appropriate Lyapunov Functional to Professor Chill.

\section{Discussion of the conjectured properties in Speetjens et al.}

Note, in the following quotes the solution of the parabolic problem is referred to as "the system":

1) [SR]: Property 1:(p.937)

"The system results in a global semiflow: $\Phi(t): H^{1}(\Omega) \rightarrow H^{1}(\Omega), t \in[0, \infty)$."

2) $[\mathrm{SR}]:$ Property 2: (p.938)

"Assume that the global semiflow $\Phi$ generated by the system has some compactness properties, e.g.: in the sense of [18, Theorem 1.1., Assmpt. (1.12) or (1.13)] and further assume the existence of a bounded set $M \subset H^{1}(\Omega)$, which attracts all bounded sets in $H^{1}(\Omega)$." 1

\footnotetext{
${ }^{1}$ There is an obvious typographical error in $[\mathrm{SR}]$ as to the number of the theorems, which we corrected in this quote. The reference [18] of [SR] refers to Temam's book [T].
} 
3) [SR]: Property 3: (p.938) (implied)

$$
E(u)=\frac{\nu}{2}\left(\int_{\Omega}|\nabla u|^{2} d \xi+\int_{S_{1}} F(u) d x\right),
$$

is a Lyapunov Functional for $\Phi$ satisfying the assumptions of [18, Theorem VII, 4.1], $S_{1}=B_{R} \times\{H\}$.

It should be noted that in [SR] the boundary Neumann boundary condition with $-u_{y}(t, x, 0)=1$ and a cubic like function $f$ as in Fig 1 . is considered. The transformation

$$
\tilde{u}=u+y+d \text {. }
$$

yields the above problem for an appropriate choice of $d$, with a modified function $f$ satisfying the assumptions given below.

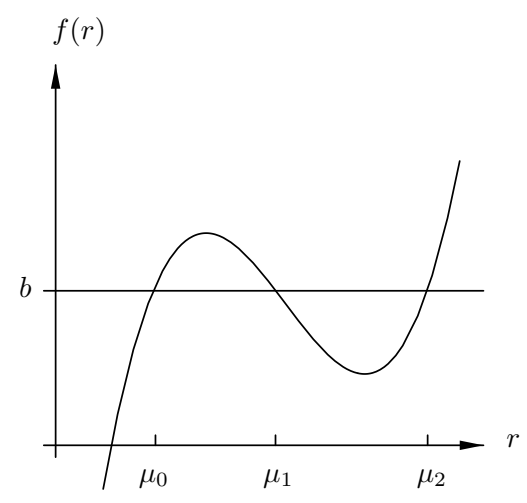

Fig. 1

Since we want to deal with solutions in Sobolev spaces we assume that $f$ has linear growth for large values of the argument. The physical relevant values of the temperature are certainly within a bounded range, hence that assumption does not restrict the applications of the model to the physical regime. To be precise we always assume that the nonlinearity $f$ satisfies

$$
\left(A_{f}\right) \begin{cases}\text { 1) } & f: \mathbb{R} \rightarrow \mathbb{R} \text { is globally Lipschitz, i.e. : } \\ & \left|f\left(r_{1}\right)-f\left(r_{2}\right)\right| \leq K_{1}\left|r_{1}-r_{2}\right|, \text { for } r_{1}, r_{2} \in \mathbb{R}, \\ 2) \quad & f(r) \text { has exactly three zeros at } 0, \mu_{1}, \mu_{2}, 0<\mu_{1}<\mu_{2} \\ 3) \quad & f(r) r>K_{2} r^{2}-m, \text { for all } r .\end{cases}
$$

for some positive constants $K_{1}, K_{2}, m$ ( $K_{1}, m$ bigger than 1 , say).

In the first part of this note we present an uniqueness and existence proof for weak solutions for initial values in $L^{2}$. Our arguments provide enough regularity to verify most hypotheses of the stability theorems presented in Temam's book [T], 
as we point out in the second part. To verify that $E(u)$ actually is a Lyapunov Functional with the required properties to apply Theorem VII, 4.1 of [T], we need some stronger estimates for which we assume that $f$ is differentiable, in addition.

Certainly, there are many results available on the solutions of (NP) in the literature. But these mostly contain various compatibility and regularity conditions on the initial value. c.f. eg. [A]. Here we point out that a straight forward Faedo-Galerkin approximation yields the existence of a unique solution without those conditions. That allows us to consider the semigroup in $L^{2}(\Omega)$, and not in $H^{1}(\Omega)$, as suggested in $[\mathrm{SR}]$.

Due to the imperfection in the material it seems advantageous to develop a theory with as little regularity in the space variables as possible. Furthermore any restrictions to the initial temperature distribution such as a-priory compatibility conditions would be hard to meet in experiments. Therefore, to deal with the semigroup in the basic Hilbert space $L^{2}(\Omega)$ is not only mathematically more appropriate but also better suited for the applications.

In order to do so we need a rather general notion of weak solutions which allows for initial values in $L^{2}(\Omega)$ :

Definition 1 Let $u:((0, \infty) \times \Omega \rightarrow \mathbb{R}$ be a (Lebesgue-) measurable function in the spaces

$$
C\left([0, \infty), L^{2}(\Omega)\right), L^{2}\left((0, N), L^{2}\left(S_{1}\right)\right), L^{2}\left((0, N), W^{1,2}(\Omega)\right)
$$

and

$$
W^{1,2}\left(\left(\frac{1}{N}, N\right) \times \Omega\right), \text { for all } N \in \mathbb{N},
$$

then we call $u$ a weak solution of (NP) for $u_{\mathrm{o}} \in L^{2}(\Omega)$, if

$$
\begin{aligned}
& -\int_{0}^{\infty} \int_{\Omega} u \phi_{t} d \xi d t+\nu\left[\int_{0}^{\infty} \int_{\Omega} D u D \phi d \xi d t+\int_{0}^{\infty} \int_{S_{1}} f(u) \phi d x d t\right] \\
& =\int_{\Omega} u_{\mathrm{o}} \phi(0) d \xi,
\end{aligned}
$$

for all functions $\phi \in C_{0}^{1}\left(\mathrm{R}, C^{\infty}(\Omega)\right)$.

Remarks:

1) Our approach is applicable for more general domains $\Omega \subset \mathbb{R}^{n}$ with a nonlinear boundary condition of the form

$$
\left.\frac{\partial}{\partial \mathbf{n}} u\right|_{\Omega}=f(x, u) .
$$

However, in order to get some rather explicit estimates we restrict the discussion to $\Omega=B_{R} \times(0, H) \subset \mathbb{R}^{n}$.

2) The assumption $u \in W^{1,2}\left(\left(\frac{1}{N}, N\right) \times \Omega\right)$, for all $N$ implies that the trace of $u$ is a measurable function in $(0, \infty) \times\left(S_{1}\right)$. Consequently $u$ is well defined in $L_{l o c}^{2}\left((0, \infty), L^{2}\left(S_{1}\right)\right)$, and $u \in L^{2}\left((0, N), L^{2}\left(S_{1}\right)\right)$ is a meaningful hypothesis. 
3) Note that our notion of solutions implies that $u(0)=u_{\mathrm{o}}$ in $L^{2}(\Omega)$. This follows from choosing testfunctions $\phi=\tilde{\phi}_{\epsilon}(t) \psi$ with $\psi \in C^{\infty}(\Omega)$ and $\tilde{\phi}_{\epsilon}$ approximating

$$
\phi_{\epsilon}= \begin{cases}1 & \text { for } t<0 \\ 1-\frac{1}{\epsilon} t & \text { for } 0 \leq t \leq \epsilon \\ 0 & \text { for } \epsilon<t\end{cases}
$$

4) Our existence proof provides the estimates for the Sobolev Spaces listed in Def. 1 , but we need to show the continuity of the solution on $[0, \infty)$, in $L^{2}(\Omega)$ separately.

We have

Theorem 1. If $f$ satisfies $\left(A_{f}\right)$, then for each $u_{\mathrm{o}} \in L^{2}(\Omega)$, there is a unique weak solution $u$ of $(\mathrm{NP})$.

We prove the theorem in the next two sections. In the last section we furnish further estimates which allow us to verify all the properties of the attractor postulated in $[\mathrm{SR}]$.

\section{Uniqueness}

Our proof is modeled after the proof presented in Sohr's book of Serrin's uniqueness result for Navier-Stokes equations in 3-D ( as long as the solutions remains smooth), c.f.: [Se, So].

Because of Gronwall's Lemma the uniqueness is a consequence of the following estimate:

For weak solutions $u, w$ we have

$$
\begin{aligned}
& \frac{1}{2} \int_{\Omega}((u-w)(T))^{2} d \xi+\nu \int_{0}^{T} \int_{\Omega}|\nabla(u-w)|^{2} d \xi d t \\
& \leq \nu\left|\int_{0}^{T} \int_{S_{1}}(f(u)-f(w))(u-w) d x d t\right| \\
& \leq \frac{\nu}{2} \int_{0}^{T} \int_{\Omega}|\nabla(u-w)|^{2} d \xi d t+C \int_{0}^{T} \int_{\Omega}(u-w)^{2} d \xi d t .
\end{aligned}
$$

With $z(s)=\int_{0}^{s} \int_{\Omega}(u-w)^{2} d \xi d t$, we have $z(0)=0$ and hence Gronwall's Lemma implies that

$$
\int_{0}^{s} \int_{\Omega}(u-w)^{2} d \xi d t=0, \text { for all } s>0
$$


We start with the justification of the last inequality of (1). For smooth functions $\tilde{u}$ and $\tilde{w}$, the compact trace of $W^{1,2}(\Omega)$ in $L^{2}\left(S_{1}\right)$ yields

$$
\begin{aligned}
& \left|\int_{\delta} \int_{S_{1}}(f(\tilde{u})-f(\tilde{w}))(\tilde{u}-\tilde{w}) d x d t\right| \\
& \leq K_{1} \int_{\delta}^{T} \int_{S_{1}}(\tilde{u}-\tilde{w})^{2} d t \\
& \leq \frac{\nu}{2} \int_{\delta}^{T} \int_{\Omega}|\nabla(\tilde{u}-\tilde{w})|^{2} d \xi d t+C \int_{\delta}^{T} \int_{\Omega}(\tilde{u}-\tilde{w})^{2} d \xi d t,
\end{aligned}
$$

with the constant $C=C\left(K_{1}, \Omega\right)$, but not depending on $0<\delta<T$.

To see that (2) holds for two solutions $u$ and $w$, we approximate the solutions with smooth functions in $W^{1,2}\left(\left(\frac{1}{N}, N\right) \times \Omega\right)$ with $\frac{1}{N}<\delta$. We note that the trace operator into $L^{2}\left(\left(\frac{1}{N}, N\right) \times S_{1}\right)$, is compact. Hence the inequality is true in the limit with the constant not depending on $\delta$.

Also, the integrability condition on the solutions together with the growth condition on $f$ provide that the two quantities

$$
\left|\int_{0}^{\delta} \int_{S_{1}}(f(u)-f(w))(u-w) d x d t\right|
$$

and

$$
\frac{\nu}{2} \int_{0}^{\delta} \int_{\Omega}|\nabla(u-w)|^{2} d \xi d t+C \int_{0}^{\delta} \int_{\Omega}(u-w)^{2} d \xi d t,
$$

can be estimated by $o(\delta)$ with $o(\delta) \rightarrow 0$, as $\delta \rightarrow 0$, establishing the second inequality of $(1)$.

For the task to verify the first inequality of (1) we furnish further properties of the solution implied by the definition. To this end let $\mu_{\epsilon}$ be a mollifier in $t$, (with support of length $2 \epsilon)$. For $t>\epsilon$ and a function $v \in L^{1}((0, \infty) \times \Omega)$ we define the partially mollified function $v_{\epsilon}$ by

$$
v_{\epsilon}(t, \xi)=\int_{0}^{\infty} v(\tau, \xi) \mu_{\epsilon}(t-\tau) d \tau
$$

where we identify $v$ with its trivial extension, when necessary.

We list some properties of that partial mollification in

Lemma A. Let $D$ be a measurable set in $\mathbb{R}^{m}$. 
1) For $v$ in $L^{2}((0, T) \times D)$ and $\delta>2 \epsilon$, we have $v_{\epsilon} \rightarrow v$ in $L^{2}((\delta, T) \times D)$, as $\epsilon \rightarrow 0$.

For weak solutions $u, w$ their mollified functions have the additional properties:

2) If $t \geq \delta>2 \epsilon$, then

$$
\int_{\Omega}\left(u_{\epsilon}(t)-u(t)\right)^{2} d \xi \rightarrow 0 \text { as } \epsilon \rightarrow 0 .
$$

3) For $t \geq \delta>2 \epsilon$, we have $u_{\epsilon} \in C^{1}\left((\delta, \infty), W^{1,2}(\Omega)\right)$, and

$$
\frac{\partial}{\partial \xi_{i}} u_{\epsilon}(t, \xi)=\int_{-\infty}^{\infty} \mu_{\epsilon}(t-\tau) \frac{\partial}{\partial \xi_{i}} u(\tau, \xi) d \tau=\left(\frac{\partial}{\partial \xi_{i}} u\right)_{\epsilon}(t, \xi) .
$$

4) For $t \geq \delta>2 \epsilon$, the function $t \rightarrow \int_{\Omega} u_{\epsilon} w_{\epsilon}(t) d \xi$ is differentiable and

$$
\frac{d}{d t} \int_{\Omega} u_{\epsilon} w_{\epsilon}(t) d \xi=\int_{\Omega} u_{\epsilon}^{\prime} w_{\epsilon}(t) d \xi+\int_{\Omega} u_{\epsilon} w_{\epsilon}^{\prime}(t) d \xi
$$

with $u_{\epsilon}^{\prime}(t, x)=\left(u_{\epsilon}\right)_{t}(t, x)=\int_{0}^{\infty} u(\tau, x)\left(\mu_{\epsilon}\right)^{\prime}(t-\tau) d \tau$.

5) For $\delta>2 \epsilon$, we have

$$
\int_{\delta}^{T} \int_{\Omega} u_{\epsilon}^{\prime} w_{\epsilon} d \xi d t=-\nu\left[\int_{\delta}^{T} \int_{\Omega} \nabla u_{\epsilon} \nabla w_{\epsilon} d \xi d t+\int_{\delta}^{T} \int_{S} f(u)_{\epsilon} w_{\epsilon} d x d t\right] .
$$

The proof of Lemma A mainly follows the basic arguments provided in [So], which we give in the appendix for the convenience of the reader.

For two solutions Lemma A implies

$$
\begin{aligned}
& \int_{\Omega} u w(T) d \xi-\int_{\Omega} u w(\delta) d \xi=\lim _{\epsilon \rightarrow 0}\left[\int_{\Omega} u_{\epsilon} w_{\epsilon}(T) d \xi-\int_{\Omega} u_{\epsilon} w_{\epsilon}(\delta) d \xi\right] \\
& =\lim _{\epsilon \rightarrow 0}\left[\int_{\delta}^{T} \int_{\Omega} u_{\epsilon}^{\prime} w_{\epsilon} d \xi d t+\int_{\delta}^{T} \int_{\Omega} w_{\epsilon}^{\prime} u_{\epsilon} d \xi d t\right] \\
& =-\nu \lim _{\epsilon \rightarrow 0}\left[\int_{\delta}^{T} \int_{\Omega} \nabla u_{\epsilon} \nabla w_{\epsilon} d \xi d t+\int_{\delta} \int_{S} f(u)_{\epsilon} w_{\epsilon} d x d t\right] \\
& -\nu \lim _{\epsilon \rightarrow 0}\left[\int_{\delta}^{T} \int_{\Omega}^{T} \nabla w_{\epsilon} \nabla u_{\epsilon} d \xi d t+\int_{\delta} \int_{S} f(w)_{\epsilon} u_{\epsilon} d x d t\right]
\end{aligned}
$$


We note that due to our assumptions the quantities

$$
\int_{0}^{\delta} \int_{\Omega} \nabla u \nabla w d \xi d t \text { and } \int_{0}^{\delta} \int_{S} f(u) w d x d t
$$

vanish as $\delta \rightarrow 0$, whereas

$$
\int_{\Omega} u w(\delta) d \xi \rightarrow \int_{\Omega} u_{\mathrm{o}}^{2} d \xi
$$

Hence

$$
\begin{aligned}
& \int_{\Omega} u w(T) d \xi-\int_{\Omega} u_{0}^{2} d \xi=-\nu\left[2 \int_{0}^{T} \int_{\Omega} \nabla w \nabla u d \xi d t\right. \\
& \left.+\int_{0}^{T} \int_{S} f(w) u d x d t+\int_{0}^{T} \int_{S} f(u) w d x d t\right] .
\end{aligned}
$$

With this equation we can verify the first inequality of (1) expanding the square under the integral on the left hand side and then choosing solutions $u$ and $w$ for each term, fittingly. We conclude that the solution has to be unique.

\section{Existence}

To establish the existence of a weak solution we use a Faedo-Galerkin approximation. As usually, for a sequence $\left(\psi_{n}\right)$ of smooth functions on $\Omega$ dense in the Sobolev space $W^{1,2}(\Omega)$, we define a finite dimensional space $V_{n}$, say, by $V_{n}=\operatorname{span}\left\{\psi_{1}, \ldots, \psi_{n}\right\}$.

A differentiable function $u_{n}(t):[0, \infty) \rightarrow V_{n}$ is called $n$-th Faedo - Galerkin approximation if it satisfies

i) $u_{n}(0)=\left(u_{n}\right)_{\mathrm{o}}$ for elements $\left(u_{n}\right)_{\mathrm{o}} \in V_{n}$ with $\left(u_{n}\right)_{\mathrm{o}} \rightarrow u_{\mathrm{o}}$ in $L^{2}(\Omega)$ as $n \rightarrow \infty$,

and

ii) $\int_{\Omega}\left(u_{n}\right)_{t} \phi d \xi+\nu\left[\int_{\Omega} D u_{n} D \phi d \xi+\int_{S_{1}} f\left(u_{n}\right) \phi d x\right]=0$,

for all functions $\phi \in V_{n}$ and $t \in[0, \infty)$.

Since $V_{n}$ is a finite dimensional (real) vector space it is isomorphic to $\mathbb{R}^{n}$. Hence we can consider $u_{n}$ as a function $u_{n}(t):[0, \infty) \rightarrow \mathrm{R}^{n}$ and rewrite the above equation in the form

$$
u_{n}^{\prime}=\mathcal{F}_{n}\left(u_{n}\right),
$$

which is a system of first order ODE's. A unique solution of the system exists, for all $t \geq 0$ provided $\mathcal{F}_{n}(r)$ is globally Lipschitz.

Since the norms on finite dimensional vector spaces are equivalent, a global Lipschitz bound can be found for each $\mathcal{F}_{n}$ because $f$ is supposed to be globally Lipschitz. 
Note also, that for fixed $t$ the approximation $u_{n}$ and its derivative $u_{n}^{\prime}$ are in $V_{n}$ and consequently those can be used as test functions in (FG). Testing with $u_{n}$ and integrating over $(0, N)$ we get

$$
\frac{1}{2} \frac{d}{d t} \int_{\Omega} u_{n}^{2}(t) d \xi+\nu\left[\int_{\Omega}\left|\nabla u_{n}\right|^{2} d \xi+\int_{S_{1}} f\left(u_{n}\right) u_{n} d x\right]=0,
$$

firstly, and then

$$
\begin{aligned}
& \int_{\Omega}\left(u_{n}\right)^{2}(N) d \xi+2 \nu\left[\int_{0}^{N} \int_{\Omega}\left|\nabla u_{n}\right|^{2} d \xi d t+\int_{0}^{N} \int_{S_{1}} f\left(u_{n}\right) u_{n} d x d t\right] \\
& =\int_{\Omega}\left(\left(u_{n}\right)_{\mathrm{o}}\right)^{2} d \xi .
\end{aligned}
$$

Due to the bounds from below on $f(r) r$ we conclude that the sequence $u_{n}$ is bounded in $L^{2}\left((0, N), W^{1,2}(\Omega)\right)$. That allows us to go to the limit in the first two (linear) terms in the definition of the weak solution, on account of the weak compactness of bounded sets in Hilbert spaces.

We also get a bound on the trace of $u_{n}$ in $L^{2}\left((0, N), L^{2}\left(S_{1}\right)\right)$, but in order to interchange the limit in the nonlinear term we need further information, such as the point wise convergence with respect to $S_{1}$. The later would follow from weaker estimates than those we furnish below. But these estimates will provide the bounds, too, which we utilize in the discussion of the properties of the semigroup for large $t$.

Firstly, we prove that there is a uniform bound on the $L^{2}(\Omega)$-norm of $u(t)$ for all $t>0$ and all $N$. We begin with an observation due to the special geometry of the domain.

Lemma B If $u \in W^{1,2}(\Omega), \Omega=B_{R} \times(0, H)$, then

$$
\|u\|_{L^{2}(\Omega)}^{2} \leq 2 H^{2} \int_{\Omega}|\nabla u|^{2} d \xi+2 H \int_{S_{1}} u^{2} d x .
$$

Proof:

For $u \in C^{1}(\bar{\Omega})$ and $\xi=(x, y) \in \Omega$ we have

$$
|u(\xi)|=\left|u(x, H)-\int_{y}^{H} u_{y}(x, s) d s\right| \leq|u(x, H)|+\int_{0}^{H}\left|u_{y}\right| d y .
$$

Squaring both sides of the equation and then integrating over $\xi$ yields

$$
\int_{\Omega} u^{2} d \xi \leq 2 H^{2} \int_{\Omega}(\nabla u)^{2} d \xi+2 H \int_{S_{1}} u^{2} d x .
$$

The statement now follows from the density of $C^{1}(\bar{\Omega})$ in $W^{1,2}(\Omega)$ and the embedding theorems. (Similar estimates can be obtained with other geometries of the heating device. But we use the Lemma to get rather explicit constants.) 
Next, Lemma B and our assumptions on $f$ imply

$$
\kappa_{1} \int_{\Omega} u_{n}^{2} d \xi \leq 2 \nu\left(\int_{\Omega}\left|\nabla u_{n}\right|^{2} d \xi+\int_{S_{1}} f\left(u_{n}\right) u_{n} d x\right)+\kappa_{2},
$$

with $\kappa_{1}=\nu\left[\max \left\{H, H^{2}\right\} \max \left\{1, \frac{1}{K_{2}}\right\}\right]^{-1}$, and $\kappa_{2}=2 \nu m \int_{S_{1}} d x$.

From (3) we get

$$
\frac{d}{d t} \int_{\Omega} u_{n}^{2} d \xi+\kappa_{1} \int_{\Omega} u_{n}^{2} d \xi \leq \kappa_{2} .
$$

We invoke the following version of Gronwall's Lemma, c.f., eg.: [T].

For integrable functions $z, g, h$, with $z^{\prime}$ being locally integrable, too, we have

$$
\begin{aligned}
& z^{\prime} \leq g z+h \Rightarrow \\
& z(t) \leq z\left(t_{0}\right) \exp \left(\int_{t_{0}}^{t} g(\tau) d \tau\right)+\int_{t_{0}}^{t} h(s) \exp \left(-\int_{t}^{s} g(\tau) d \tau\right) d s .
\end{aligned}
$$

With $g \equiv-\kappa_{1}$ it provides the bound

$$
\begin{aligned}
& \int_{\Omega} u^{2}(t, \xi) d \xi \leq e^{-\kappa_{1} t} \int_{\Omega} u_{0}^{2} d \xi+\frac{\kappa_{2}}{\kappa_{1}}\left(1-e^{-\kappa_{1} t}\right) \\
& \leq \int_{\Omega} u_{0}^{2} d \xi+\frac{\kappa_{2}}{\kappa_{1}} .
\end{aligned}
$$

In order to get an estimate on the $L^{2}((0, T) \times \Omega)$-norm of $u^{\prime}$, too, we use $u_{n}^{\prime}$ as a test function in the definition of the weak solution. Since the initial value is not in $W^{1,2}(\Omega)$, a straight forward integration over the second term does not provide the required bound. Instead we use "Uniform Gronwall Lemma" which is attributed by Temam to Foias and Prodi [FP], c.f.: [T], p.91.

For locally integrable, nonnegative functions $z, g, h$, with $z^{\prime}$ being locally integrable, too, we have that

$$
z^{\prime} \leq g z+h
$$

implies

$$
z(t+s) \leq\left(z\left(t_{1}\right)+\int_{t_{1}}^{t+s} h(\tau) d \tau\right) \exp \left(\int_{t_{1}}^{t+s} g(\tau) d \tau\right)
$$

and

$$
z(t+s) \leq\left[\frac{1}{s} \int_{t}^{t+s} z(\tau) d \tau+\int_{t}^{t+s} h(\tau) d \tau\right] \exp \left(\int_{t}^{t+s} g(\tau) d \tau d s\right)
$$


for $0 \leq t_{0} \leq t_{1}<t+s$.

The point being here is that the last estimate does not need any information on $z$ at the initial boundary point of the integration interval.

To use this fact, we need to control the terms on the right hand side:

Identity (3) and the assumptions on $f$ also provide the estimate

$$
\frac{d}{d t} \int_{\Omega} u_{n}^{2}(t) d \xi+2 \nu \int_{\Omega}\left|\nabla u_{n}(t)\right|^{2} d \xi+\frac{2 \nu}{K_{2}} \int_{S_{1}} u_{n}^{2}(t) d x \leq \kappa_{2}
$$

Integrating over $(t, t+s)$ we get

$$
\begin{aligned}
& \nu \int_{t}^{t+s} \int_{\Omega}\left|\nabla u_{n}\right|^{2} d \xi d \tau+\nu \int_{t}^{t+s} \int_{S_{1}} u_{n}^{2} d x d \tau \leq s \frac{\bar{K}_{2}}{2} \kappa_{2}+\frac{\bar{K}_{2}}{2} \int_{\Omega} u_{n}^{2}(t) d \xi \\
& \leq s \frac{\bar{K}_{2}}{2} \kappa_{2}+\tilde{\rho},
\end{aligned}
$$

with $\bar{K}_{2}=\max \left\{1, \frac{1}{K_{2}}\right\}$, and

$$
\tilde{\rho}=\frac{\bar{K}_{2}}{2} \int_{\Omega} u_{n}^{2}(t) d \xi \leq \frac{\bar{K}_{2}}{2}\left(\int_{\Omega} u_{0}^{2} d \xi+\frac{\kappa_{2}}{\kappa_{1}}\right)=: \tilde{\rho}_{0} \text {, because of }(5) .
$$

Now let $F$ be the smallest nonnegative antiderivative of $f$, then testing (FG) with $u_{n}^{\prime}$ yields

$$
\frac{d}{d t}\left[\int_{\Omega}\left|\nabla u_{n}\right|^{2} d \xi+\int_{S_{1}} F\left(u_{n}\right) d x\right] \leq 0 .
$$

To use the uniform Gronwall Lemma for

$$
z=\int_{\Omega}\left|\nabla u_{n}\right|^{2} d \xi+\int_{S_{1}} F\left(u_{n}\right) d x
$$

we need to estimate the second term. From the growth condition on $f$ we get that $F(s) \leq c\left(s^{2}+1\right)$, for some $c \geq 1 .\left(c \geq \max \left\{1, \frac{1}{2} K_{1}\left(1+\mu_{2}^{2}\right)\right\}\right.$ will do. $)$ Hence

$$
\begin{aligned}
& \int_{t}^{t+s} z d \tau \leq \int_{t}^{t+s} \int_{\Omega}\left|\nabla u_{n}\right|^{2} d \xi d \tau+c\left(s^{2}+1\right) \int_{t}^{t+s} \int_{S_{1}} u_{n}^{2} d x d \tau \\
& \leq \frac{c}{\nu}\left(s^{2}+1\right)\left(s \frac{\bar{K}_{2}}{2} \kappa_{2}+\tilde{\rho}\right) .
\end{aligned}
$$

With $h=g=0$ and $s \leq 1$ we get from the uniform Gronwall Lemma that

$$
\int_{\Omega}\left|\nabla u_{n}(s)\right|^{2} d \xi+\int_{S_{1}} F\left(u_{n}(s)\right) d x \leq \frac{\kappa_{3}}{s},
$$


for $\kappa_{3}=\frac{2 c}{\nu}\left(\frac{\bar{K}_{2}}{2} \kappa_{2}+\tilde{\rho}_{0}\right)$.

Next, integrating $(\mathrm{FG}) \operatorname{over}\left(\frac{1}{N}, N\right)$, with the testfunction $u_{n}^{\prime}$ yields

$$
\int_{1 / N}^{N} \int_{\Omega}\left(u_{n}^{\prime}\right)^{2} d \xi d t \leq \int_{\Omega}\left|\nabla u_{n}\left(\frac{1}{N}\right)\right|^{2} d \xi+\int_{S_{1}} F\left(u\left(\frac{1}{N}\right)\right) d x \leq \kappa_{3} N .
$$

and hence $u_{n}$ is bounded in $W^{1,2}\left(\left(\frac{1}{N}, N\right) \times \Omega\right)$ for each each $N$.

Now we can verify that the limit $u$ of the sequence of Garlerkin solutions is indeed a weak solution. From the bounds established above we conclude that (a subsequence of ) $u_{n}$ converges to $u$ weakly in the Hilbert spaces $L^{2}\left((0, N), W^{1,2}(\Omega)\right)$ and $W^{1,2}\left(\left(\frac{1}{N}, N\right) \times \Omega\right)$, for all $N$.

Hence $u_{n}$ converges strongly in $L^{2}\left(\left(\frac{1}{N}, N\right) \times S_{1}\right)$, for all $N$. As a consequence we can assume that $u_{n}(t, x) \rightarrow u(t, x)$, a.e. in $(0, \infty) \times S_{1}$, (choosing a further subsequence if necessary). Because we have a bound on the trace of $u_{n}$ in $L^{2}\left((0, N) \times S_{1}\right)$ the growth condition on $f$ implies the weak convergence of $f\left(u_{n}\right)$ to $f(u)$ in that space; consequently $u$ satisfies the defining equation of a weak solution.

Altogether $u_{n}$ is bounded in $L^{\infty}\left((0, \infty), L^{2}(\Omega)\right), L^{2}\left((0, N), L^{2}\left(S_{1}\right)\right)$, and $L^{2}\left((0, N), W^{1,2}(\Omega)\right)$ for all $N$, hence the limit function $u$ is also in those spaces.

It remains to verify the continuity of $u:[0, \infty) \rightarrow L^{2}(\Omega)$. We note that for all $t>0$ the norm $\|u(t)\|_{L^{2}(\Omega)}$ is well defined: Because of the compact trace operator

$$
\begin{aligned}
& t r: W^{1,2}\left(\left(\frac{1}{N}, N\right) \times \Omega\right) \rightarrow L^{2}(\{t\} \times \Omega), \text { for } t \in\left(\frac{1}{N}, N\right), \text { we have } \\
& u_{n}(t) \rightarrow u(t), \text { in } L^{2}(\Omega) \text {, as } n \rightarrow \infty .
\end{aligned}
$$

We begin with verifying the weak continuity at $t=0$. For test functions $\phi$ we have

$$
\begin{aligned}
& \left|\int_{\Omega}\left(u(\tau)-u_{\mathrm{o}}\right) \phi d \xi\right|=\left|\lim _{n \rightarrow \infty} \int_{\Omega}\left(u_{n}(\tau)-\left(u_{n}\right)_{\mathrm{o}}\right) \phi d \xi\right| \\
& =\left|\lim _{n \rightarrow \infty} \int_{0}^{\tau} \int_{\Omega} u_{n}^{\prime}(t) \phi d \xi d t\right| \leq \lim _{n \rightarrow \infty} \omega(\phi, \tau)\left[\left(\int_{0}^{\tau} \int_{\Omega}\left|\nabla u_{n}\right|^{2} d \xi d t\right)^{1 / 2}\right. \\
& \left.+\left(\int_{0}^{\tau} \int_{S_{1}} u_{n}^{2} d x d t\right)^{1 / 2}\right] \leq C \omega(\phi, \tau)
\end{aligned}
$$

with $\omega(\phi, \tau) \rightarrow 0$, as $\tau \rightarrow \infty$.

Since $L^{2}(\Omega)$ is a Hilbert space, the strong continuity at zero now follows from the estimate 


$$
\begin{aligned}
& \int_{\Omega}(u(\tau))^{2} d \xi-\int_{\Omega}\left(u_{\mathrm{o}}\right)^{2} d \xi \\
& =\lim _{n \rightarrow \infty} \int_{\Omega}\left(u_{n}(\tau)\right)^{2} d \xi-\int_{\Omega}\left(\left(u_{n}\right)_{\mathrm{o}}\right)^{2} d \xi \\
& =\frac{1}{2} \lim _{n \rightarrow \infty} \int_{0}^{\tau} \int_{\Omega}\left(u_{n}^{\prime} u_{n}\right) d \xi d t \leq \lim _{n \rightarrow \infty}-\int_{0}^{\tau} \int_{S_{1}} f\left(u_{n}\right) u_{n} d x d t \\
& \leq C \tau \int_{S_{1}} d x
\end{aligned}
$$

the last inequality holds because of the bound from below on $f(r) r$.

To verify the continuity for $t>0$ we note that the compact trace operator ensures that for two given $t_{1}, t_{2}$ there is a subsequence such that $u_{n}\left(t_{i}\right)$ converges strongly in $L^{2}(\Omega)$. So

$$
\left\|u\left(t_{1}\right)-u\left(t_{2}\right)\right\|_{2}=\lim _{n \rightarrow \infty}\left\|u_{n}\left(t_{1}\right)-u_{n}\left(t_{2}\right)\right\|_{2} .
$$

On the other hand Jensen's inequality allows the following estimate

$$
\begin{aligned}
& \left\|u_{n}\left(t_{1}\right)-u_{n}\left(t_{2}\right)\right\|_{2}^{2}=\int_{\Omega}\left(\int_{t_{1}}^{t_{2}}\left(u_{n}^{\prime}(t) d t\right)^{2} d \xi\right. \\
& \leq\left|\int_{\Omega}\right| t_{1}-t_{2}\left|\int_{t_{1}}^{t_{2}}\left(u_{n}^{\prime}(t)\right)^{2} d t d \xi \leq\right| t_{1}-t_{2} \mid \int_{1 / N}^{N} \int_{\Omega}\left(u_{n}^{\prime}(t)\right)^{2} d \xi d t \\
& \leq\left|t_{1}-t_{2}\right| c(N) .
\end{aligned}
$$

for a fixed $N, 0<\frac{1}{N}<t_{1}, t_{2}<N$, (independently of $n$, ) implying the continuity of the solution in $L^{2}(\Omega)$.

That concludes the existence proof.

\section{Properties of the semigroup}

Firstly we note that Theorem 1 provides the existence of a continuous semigroup:

Theorem 2 A weak solutions of (NP) generates a continuous semigroup $\Phi(t): L^{2}(\Omega) \rightarrow L^{2}(\Omega) ; u_{o} \rightarrow u(t)$, i.e.:

i) $\left\{\begin{array}{l}\Phi(t+s)=\Phi(t) \circ \Phi(s) \quad \text { for } s, t>0, \\ \Phi(0)=i d_{L^{2}(\Omega)}\end{array}\right.$

ii) $\Phi(t)$ is continuous in $L^{2}(\Omega)$. 
Further properties are obtained immediately from the estimates in the existence proof, c.f.: $[\mathrm{T}$, p.85,ff], too. Inequality (4) shows

Corollary 1 Every ball $B(0, \rho) \subset L^{2}(\Omega)$ with $\rho^{2}>\frac{\kappa_{2}}{\kappa_{1}}$ is an absorbing set.

More precisely we get from (5), that if $u_{\mathrm{o}}(x) \in B(0, r) \subset L^{2}(\Omega)$ then with $r>\rho>$ $\rho_{0}=\sqrt{\frac{\kappa_{2}}{\kappa_{1}}}$, we have

$$
\int_{\Omega} u^{2}(t, \xi) d \xi \leq \rho_{0}^{2}+e^{-\kappa_{1} t}\left(r^{2}-\rho_{0}^{2}\right) .
$$

So if $t \geq \frac{1}{\kappa_{1}} \ln \left(\frac{r^{2}-\rho_{0}^{2}}{\rho^{2}-\rho_{0}^{2}}\right):=t_{r, \rho}$, then

$$
\int_{\Omega} u^{2}(t, \xi) d \xi \leq \rho^{2}
$$

and $u(t) \in B(0, \rho) \subset L^{2}(\Omega)$.

Actually we have shown the estimates only for the Faedo- Galerkin approximations $u_{n}$. Because of the lower semi-continuity properties of the norm involved these are valid for the limit, also.

In order to apply the theory of the existence of an attractor we need that the image of a ball lies in a compact set of $L^{2}(\Omega)$.

Corollary 2 If $u_{\mathrm{o}}(x) \in B(0, r) \subset L^{2}(\Omega)$ then for $t>t_{r, \rho}+1$ we have

$$
u(t) \in B(0, R) \subset W^{1,2}(\Omega)
$$

for $R^{2}>\kappa_{3}+\rho^{2}$, and $\rho>\rho_{0}$.

To see that, we note that from Corollary 1 and inequality (6) we get

$$
\left(\|u\|_{1,2}\right)^{2} \leq \rho^{2}+\int_{\Omega}|\nabla u(t)|^{2} d \xi+\int_{S_{1}} F(u(t)) d x \leq R^{2} .
$$

To show that the energy functional $E(u)$ can be considered as a Lyapunov Functional on an appropriate subset we need further estimates. To get those we assume that $f$ is continuously differentiable. The global Lipschitz condition then implies that the derivative is bounded by $K_{1}$.

Testing (FG) with $u_{n}^{\prime}$ and integrating over $(t, t+1), t>t_{r,(\rho+1)}$ also gives 


$$
\int_{t}^{t+1} \int_{\Omega}\left(u_{n}^{\prime}\right)^{2} d \xi d t \leq \kappa_{3} .
$$

Hence differentiating (FG) and multiplying with $u_{n}^{\prime}$ we get:

$$
\begin{aligned}
& \frac{1}{2} \frac{d}{d t} \int_{\Omega}\left(u_{n}^{\prime}\right)^{2} d \xi+\nu \int_{\Omega}\left|\nabla u_{n}^{\prime}\right|^{2} d \xi=-\nu \int_{S_{1}} f^{\prime}\left(u_{n}\right)\left(u_{n}^{\prime}\right)^{2} d x \\
& \leq \frac{\nu}{2} \int_{\Omega}\left|\nabla u_{n}^{\prime}\right|^{2} d \xi+C \int_{\Omega}\left(u_{n}^{\prime}\right)^{2} d \xi,
\end{aligned}
$$

and now the uniform Gronwall Lemma provides the estimate

$$
\left\|u_{n}^{\prime}(t+1)\right\|_{L^{2}(\Omega)}^{2} \leq \kappa_{3} e^{2 C}:=\kappa_{4} .
$$

Consequently, for every $t>t_{r, \rho_{0}}+1$ the sequence $u_{n}^{\prime}(t)$ converges to a function $u^{\prime}(t)=v$, weakly in $W^{1,2}(\Omega)$, with $v$ being a weak solution of

$$
\left\{\begin{array}{l}
\Delta v=g, \\
\frac{\partial}{\partial n} v=h,
\end{array}\right.
$$

where $g \in L^{2}$ and $h \in L^{q}$ with $q=q(n)>2$. For $n=3$ we have $q=4$.

Invoking now the results of $[\mathrm{Gr}]$ and $[\mathrm{JK}]$ we obtain

Corollary 3 If $u_{\mathrm{o}}(x) \in B(0, r) \subset L^{2}(\Omega)$ then for $t>t_{r, \rho_{0}}+1$ we have

$$
u(t) \in B(0, \mathcal{R}) \subset H^{3 / 2}(\Omega),
$$

for some number $\mathcal{R}<\infty$.

With the definitions given in $[\mathrm{T}]$ these results imply that the semigroup $\Phi$ generated by the weak solutions of (NB) satisfies the first two properties stated in [SR].

\section{Theorem 3}

1) Every ball $B(0, \rho) \subset L^{2}(\Omega)$, is an absorbing set for $\rho>\rho_{0}(f, H)$,

2) The semigroup $\Phi$ is uniformly compact. i.e:

There is a number $t_{0}(\mathcal{B})>0$ such that

$$
\bigcup_{t \geq t_{0}} \Phi(t) \mathcal{B}
$$

is relative compact in $L^{2}(\Omega)$ for all bounded sets $\mathcal{B}$.

3) $\Phi$ has a compact connected attractor $\mathcal{A} \subset B(0, R) \subset W^{1,2}(\Omega)$ i.e.:

i) $\Phi(t) \mathcal{A}=\mathcal{A}$, for all $t \geq 0$,

ii) $\operatorname{dist}\{\Phi(\mathrm{t}) v, \mathcal{A}\} \rightarrow 0$, as $t \rightarrow \infty$, for all $v \in L^{2}(\Omega)$. 
Proof:

Corollary 2 provides the uniform compactness and then Theorem 1.1 in Chap. I of [T] establishes 3 .

To provide further properties of the attractor we consider the functional

$$
E(u(t))=\frac{\nu}{2}\left(\int_{\Omega}|\nabla u|^{2}(t) d \xi+\int_{S_{1}} F(u(t)) d x\right) .
$$

We claim that $E$ is a Lyapunov function of $\Phi$ i.e.:

There is a set $\mathcal{F} \subset L^{2}(\Omega)$ such that $E: \mathcal{F} \rightarrow \mathbb{R}$ is continuous and

1) For any $v \in \mathcal{F}$ the function $t \mapsto E(\Phi(t) v)$ is decreasing.

2) If $E(\Phi(t) v)=E(v)$ for some $t>0$, then $v$ is a fixed point of $\Phi$.

Theorem 4 In addition to $\left(A_{f}\right)$, let $f$ be continuously differentiable. Then the attractor $\mathcal{A} \subset \mathcal{F}$, with $\mathcal{F}=B(0, \mathcal{R}) \subset H^{3 / 2}(\Omega)$, and $E$ is a Lyapunov Functional on $\mathcal{F}$.

Proof:

To verify that $E(u)$ is decreasing in $t$, we can not use the Galerkin approximations directly. We have $u_{n}(t)$ is bounded in $W^{1,2}(\Omega)$ with weak limit $u(t)$, but we don't know whether it converges in the strong topology of that space. Therefore we select a sequence $\left(v_{n}\right)_{\mathrm{o}} \in V_{n}$ converging strongly to $u\left(t_{0}\right) \in W^{1,2}(\Omega)$ and consider the Galerkin solutions of $(\mathrm{NB})$ in the interval $\left(t_{0}, t\right)$ with initial value $\left(v_{n}\right)_{\mathrm{o}}$.

Because of the uniqueness theorem the solutions $\left(v_{n}\right)(t)$ converges to $u(t)$ with the additional feature that $\nabla\left(v_{n}\right)\left(t_{0}\right)$ converges to $\nabla u\left(t_{0}\right)$ and that $F\left(v_{n}(t)\right)$ converges $F(u(t))$, for $t \geq 0$, in the strong $L^{2}$-topology. Using $v_{n}^{\prime}$ as a test function we get

$$
\begin{aligned}
& \int_{\Omega}|\nabla u(t)|^{2} d \xi+\int_{S_{1}} F(u(t)) d x \\
& \leq \lim _{n \rightarrow \infty} \int_{\Omega}\left|\nabla v_{n}(t)\right|^{2} d \xi+\lim _{n \rightarrow \infty} \int_{S_{1}} F\left(u_{n}(t)\right) d x \\
& \leq \lim _{n \rightarrow \infty} \int_{\Omega}\left|\nabla v_{n}\left(t_{0}\right)\right|^{2} d \xi+\lim _{n \rightarrow \infty} \int_{S_{1}} F\left(u_{n}\left(t_{0}\right)\right) d x \\
& +\limsup _{n \rightarrow \infty}\left(-\int_{t_{0}}^{t} \int_{\Omega}\left(v_{n}^{\prime}\right)^{2} d \xi d \tau\right) \\
& \leq \int_{\Omega}\left|\nabla u\left(t_{0}\right)\right|^{2} d \xi+\int_{S_{1}} F\left(u\left(t_{0}\right)\right) d x,
\end{aligned}
$$

i.e.: $E(u)$ is decreasing. 
To verify the continuity of $\Phi$ on $\mathcal{F}$ with respect to the $L^{2}$-topology, we note that the typologies induced by $L^{2}(\Omega)$, respectively $W^{1,2}(\Omega)$, on sets $\mathcal{F}$ bounded in $H^{3 / 2}(\Omega)$ are the same. That is a consequence of the compact embedding of $H^{3 / 2}(\Omega)$ in $W^{1,2}(\Omega)$ and the compact embedding from $W^{1,2}(\Omega)$ into $L^{2}(\Omega)$ :

Obviously, we have $\|u\|_{2} \leq\|u\|_{1,2}$, so we only need to verify that we can make on $B(0, \mathcal{R}) \subset H^{3 / 2}(\Omega)$ the $W^{1,2}$-norm small provided the $L^{2}$-norm is small enough. Indeed, for given $\epsilon>0$ and for all $v, w \in \mathcal{F}=B(0, \mathcal{R}) \subset H^{3 / 2}(\Omega)$ we have

$$
\|v-w\|_{1,2}<\frac{\epsilon}{4 \mathcal{R}} 2 \mathcal{R}+C(\epsilon)\|v-w\|_{2},
$$

because of the compact embedding of $H^{3 / 2}(\Omega)$ in $W^{1,2}(\Omega)$. Then, if

$$
\|v-w\|_{2}<\delta=\frac{\epsilon}{2 C(\epsilon)},
$$

we get the desired estimate.

Theorem 4.1, Chap VII of [T] now establishes the claimed properties of the attractor:

Corollary 4 If $\mathcal{G}$ is the set of fixed points of $\Phi$, then

$$
\mathcal{A}=\mathcal{M}_{+}(\mathcal{G}) \text {. }
$$

Furthermore if $\mathcal{G}$ is discrete then $\mathcal{A}$ is the union of $\mathcal{G}$ and the heteroclinic curves joining two points of $\mathcal{G}$.

Here $\mathcal{M}_{+}(\mathcal{G})$ is the unstable manifold of $\mathcal{G}$ with respect to $\Phi$. That is the sets of all points $v$ such that for all $t \leq 0$ there is a $u(t) \in \Phi(-t)^{-1} v$, and $u(t) \rightarrow z \in \mathcal{G}$ as $t \rightarrow-\infty$, c.f.: [T], pp $489 \mathrm{ff}$.

\section{Appendix}

Proof of Lemma A:

Firstly we note that for $D \subset \mathbb{R}^{m}$ and integrable functions $v$ in $L^{1}(\mathbb{R} \times D)$ the function

$$
v_{\epsilon}(t, \xi)=\int_{0}^{\infty} v(\tau, \xi) \mu_{\epsilon}(\tau-t) d \tau,
$$

is integrable in $L^{1}(\mathbb{R} \times D)$ : Because the integrant is measurable in $\mathbb{R} \times \mathbb{R} \times D$ as the product of two measurable function, we get

$$
\begin{aligned}
& \int_{0}^{\infty} \int_{D} \int_{0}^{\infty}\left|v(\tau, \xi) \mu_{\epsilon}(\tau-t)\right| d \tau d \xi d t \\
& =\int_{0}^{\infty} \int_{0}^{\infty} \int_{D}\left|v(\tau, \xi) \mu_{\epsilon}(\tau-t)\right| \xi d \tau d t
\end{aligned}
$$




$$
\leq C(\epsilon)\|v\|_{L^{1}((0, \infty) \times \Omega)}
$$

by Tonelli's - theorem, and so $v(\tau, \xi) \mu_{\epsilon}(\tau-t)$ is in $L^{1}(\mathbb{R} \times \mathbb{R} \times D)$. Now we can ably Fubini's theorem to conclude that $v_{\epsilon}(t, \xi)$ is in $L^{1}(\mathbb{R} \times D)$.

Proof of Lemma A 1)

To verify the convergence of the mollified function in $L^{2}((\delta, T) \times D)$ note that the mollification is decreasing the norm: Due to Jensens Inequality we have

$$
\left\|u_{\epsilon}\right\|^{2} \leq\|u\|^{2} \text {. }
$$

For continuous functions $u$ a similar argument as in 2) below yields $\left(u_{n}\right)_{\epsilon} \rightarrow u_{n}$. For integrable functions there are continuous functions $u_{n}$ such that $u_{n} \rightarrow u$ in $L^{2}(\mathcal{D}), \mathcal{D}=(0, T) \times D$. So for an arbitrarily chosen $\gamma>0$ we firstly find a large enough index $n$ such that the norm of the difference $\left\|u_{n}-u\right\|_{L^{2}(\mathcal{D})}$ is less than $\frac{\gamma}{3}$, and we can choose an $\epsilon$ small enough such that $\left\|\left(u_{n}\right)_{\epsilon}-u_{n}\right\|_{L^{2}(\mathcal{D})}<\frac{\gamma}{3}$. We get

$$
\begin{aligned}
& \left\|u_{\epsilon}-u\right\|_{L^{2}(\mathcal{D})} \\
& \leq\left\|\left(u-u_{n}\right)_{\epsilon}\right\|_{L^{2}(\mathcal{D})}+\left\|\left(u_{n}\right)_{\epsilon}-u_{n}\right\|_{L^{2}(\mathcal{D})}+\left\|u_{n}-u\right\|_{L^{2}(\mathcal{D})}<\gamma .
\end{aligned}
$$

Proof of Lemma A, 2).

With Jensens inequality we get the strong convergence of the mollified weak solution in $L^{2}(\Omega)$ :

$$
\begin{aligned}
& \int_{\Omega}\left(u_{\epsilon}(t)-u(t)\right)^{2} d \xi=\int_{\Omega}\left(\int_{-\infty}^{\infty} \mu_{\epsilon}(t-\tau)(u(\tau)-u(t)) d \tau\right)^{2} d \xi \\
& \leq \int_{\Omega} \int_{-\infty}^{\infty} \mu_{\epsilon}(t-\tau)(u(\tau)-u(t))^{2} d \tau d \xi \\
& \leq \int_{-\infty}^{\infty} \mu_{\epsilon}(t-\tau) \int_{\Omega}(u(\tau)-u(t))^{2} d \xi d \tau \\
& \leq \sup _{|\tau-t|<\epsilon}(u(\tau)-u(t))^{2} d \xi \rightarrow 0,
\end{aligned}
$$

as $\epsilon \rightarrow 0$, because $u(t)$ is continuous in $L^{2}(\Omega)$, by assumption.

Proof of Lemma A, 3).

For $\phi \in C_{0}^{\infty}(\Omega)$, we have $\mu_{\epsilon} \phi \in C_{0}^{\infty}((0, \infty) \times \Omega)$, and starting with the right hand side of the equation we get 


$$
\begin{aligned}
& \int_{\Omega} \int_{-\infty}^{\infty} \mu_{\epsilon}(t-\tau) \frac{\partial}{\partial \xi_{i}} u(\tau, \xi) d \tau \phi(\xi) d \xi \\
& =\int_{-\infty}^{\infty} \int_{\Omega} \frac{\partial}{\partial \xi_{i}} u(\tau, \xi) \mu_{\epsilon}(t-\tau) \phi(\xi) d \xi d \tau \\
& =-\int_{-\infty}^{\infty} \int_{\Omega} \mu_{\epsilon}(t-\tau) u(\tau, \xi) \frac{\partial}{\partial \xi_{i}} \phi(\xi) d \xi d \tau \\
& =-\int_{\Omega} \int_{-\infty}^{\infty} \mu_{\epsilon}(t-\tau) u(\tau, \xi) d \tau \frac{\partial}{\partial \xi_{i}} \phi(\xi) d \xi .
\end{aligned}
$$

Proof of Lemma A, 4). We note that

$$
\begin{aligned}
& \frac{1}{h} \int_{\Omega}\left[u_{\epsilon}(t+h)-u_{\epsilon}(t)\right] w_{\epsilon}(t+h) d \xi \\
& =\frac{1}{h} \int_{\Omega}\left[\int_{-\infty}^{\infty} \mu_{\epsilon}(t+h-\tau) u(\tau)-\mu_{\epsilon}(t-\tau) u(\tau) d \tau\right]\left(w_{\epsilon}(t+h)\right) d \xi \\
& =\int_{\Omega}\left[\int_{-\infty}^{\infty} \mu_{\epsilon}^{\prime}(t-\tau) u(\tau) d \tau\right]\left(w_{\epsilon}(t+h)\right) d \xi \\
& +\int_{\Omega}\left[\int_{t-\epsilon}^{t+\epsilon} \omega(h, t, \tau) u(\tau) d \tau\right]\left(w_{\epsilon}(t+h)\right) d \xi \\
& \rightarrow \int_{\Omega} u_{\epsilon}^{\prime}(t)\left(w_{\epsilon}(t)\right) d \xi
\end{aligned}
$$

as $h \rightarrow 0$, since $w_{\epsilon}$ is continuous in $L^{2}(\Omega)$, and $\omega(h, t, \tau) \rightarrow 0$, uniformly in $t$ and $\tau$, as $h \rightarrow 0$. Now we can argue as in the usual proof of the product rule.

Proof of Lemma A, 5).

Firstly we note:

Due to the properties of the weak solutions, the usual completion argument provides that we can use $\phi(t) v(\xi)$ as a testfunction in the definition of weak solution, for any $v \in W^{1,2}(\Omega)$ and any smooth function $\phi$ with compact support in $(0, \infty)$, in particular for $\left.\mu_{\epsilon}^{\prime}(s-t) w_{\epsilon}(s, \xi)\right)$, with $s>\epsilon$.

For $\delta>2 \epsilon$, we have 


$$
\begin{aligned}
& \int_{\delta}^{T} \int_{\Omega} u_{\epsilon}^{\prime} w_{\epsilon} d \xi d t \\
& =\int_{\delta} \int_{\Omega} \int_{-\infty}^{\infty} u(\tau, \xi) \mu_{\epsilon}^{\prime}(t-\tau) d \tau w_{\epsilon}(t, \xi) d \xi d t \\
& =\int_{\delta} \int_{0}^{T} \int_{\Omega}^{\infty} u(\tau, \xi) \mu_{\epsilon}^{\prime}(t-\tau) w_{\epsilon}(t, \xi) d \xi d \tau d t \\
& =\int_{\delta}^{T}\left[\nu \int_{0}^{\infty} \int_{\Omega} \nabla u(\tau, \xi) \nabla\left(\mu_{\epsilon}(t-\tau) w_{\epsilon}(t, \xi)\right) d \xi d \tau\right. \\
& \left.+\int_{0}^{\infty} \int_{S} f(\tau, x)\left(t r\left(\mu_{\epsilon}(t-\tau) w_{\epsilon}(t)\right)(x)\right) d x d \tau\right] d t \\
& =\nu\left[\int_{\delta}^{T} \int_{\Omega} \nabla u_{\epsilon} \nabla w_{\epsilon} d \xi d t+\int_{\delta}^{T} \int_{S} f(u)_{\epsilon} w_{\epsilon} d x d t\right] \\
& \left.\rightarrow \int_{\delta}^{T} \int_{\Omega}^{T} \nabla u \nabla w d \xi d t+\int_{\delta}^{T} \int_{S} f(u) w d x d t\right] .
\end{aligned}
$$

To justify the limit we note that for $\frac{1}{N}<\delta-2 \epsilon$, we have that the function $u(\tau, x) \in W^{1,2}\left(\left(\frac{1}{N}, T\right) \times \Omega\right)$. Furthermore $f$ is globally Lipschitz, hence the trace operator commutes with $f$ and we get

$$
\begin{aligned}
& \int_{\delta}^{T} \int_{0}^{\infty} \int_{S} f(u(\tau, x))\left(t r\left(\mu_{\epsilon}(t-\tau) w_{\epsilon}(t)\right)(x)\right) d x d \tau d t \\
& =\int_{\delta}^{T} \int_{S}\left[\int_{0}^{\infty} f(\tau, x) \mu_{\epsilon}(t-\tau) d \tau\right] \operatorname{tr}\left(w_{\epsilon}(t)\right)(x) d x d t \\
& =\int_{\delta}^{T} \int_{S} f(u)_{\epsilon} w_{\epsilon} d x d t .
\end{aligned}
$$

Therefore all statements of Lemma 5 are verified. 


\section{References}

[A] H. Amann, Quasilinear parabolic systems under nonlinear boundary conditions. Archive for Rational Mechanics and Analysis. 92 (1986), pp. 153-192.

[AM] H. Auracher, W. Marquardt, M. Buchholz, R. Hohl, T. L ťtich, J. Blum, New Experimental Results on Steady-state and Transient Pool Boiling Heat. Transfer. Therm. Sci. Engng. 9, No. 5 (2001), 29-39.

[FP] C. Foias, and G. Prodi, Sur le comportement global des solutions non stationnaires des équations de Navier-Stokes en dimension 2. Rend. Sem. Mat. Univ. Padova, 39 (1967), 1-34.

[GS] R. W. van Gils, M. F. M. Speetjens, H. J. Zwart, H. Nijmeijer, Feedback stabilisation of a two-dimensional pool-boiling system by modal control. International Journal of Thermal Sciences, 61 (2012), 38-49.

[Gr] P. Grisvard, Smoothness of the solution of a monotonic boundary value problem for a second order elliptic equation in a general convex domain. Lecture notes in Math. 564, 135 - 151.

[JK] D. S. Jerison, C. E. Kenig, The Neumann Problem on Lipschitz Domains.Bull. AMS 4, 1981, (203-207).

[Se] J. Serrin, The initial value problem for the Navier-Stokes equations. Uni.Wisconsin Press, Nonlinear problems, Ed. R.E. Langer 1963

[So] H. Sohr, The Navier-Stokes Equations, An Elementary Functiional Analytic Approach. Basel, 2001, (Birkhaeuser).

[Sp] M. Speetjens, Steady-State Behavior of a Three-Dimensional Pool-Boiling System. Journal of Electronical Packaging. ASME 2008, (041102-1 - 041102-6).

[SR] M. Speetjens, A. Reusken, S. Maier-Paape, W. Marquardt, Stability Analysis of Two-Dimensional Pool-Boiling Systems. Siam J. Applied Dynamical Systems. 7, 2008, (933 - 961).

[T] R. Temam, Infinite-Dimensional Dynamical Systems in Mechanics and Physics. 2.ed. New York, 1997 (Springer).

Department of Mathematics, University of Oklahoma, Norman OK 73019

E-mail address: rlandes@math.ou.edu 